\title{
High-order Non-reflecting Boundary Conditions for Dispersive Waves in Cartesian, Cylindrical and Spherical Coordinate Systems
}

\author{
VINCE VAN JOOLEN ${ }^{\mathrm{a}, *}$, DAN GIVOLI ${ }^{\mathrm{b}, \dagger}$ and BENY NETA ${ }^{\mathrm{a}, \ddagger}$ \\ ${ }^{\mathrm{a}}$ Department of Mathematics, Naval Postgraduate School, 1141 Cunningham Road, Monterey, CA 93943, USA; ${ }^{\mathrm{b}}$ Department of Aerospace Engineering \\ and Asher Center for Space Research, Technion-Israel Institute of Technology, Haifa 32000, Israel
}

(Received 28 February 2003)

Dedicated to Professor Mutsuto Kawahara on the occasion of his 60th birthday

\begin{abstract}
Among the many areas of research that Professor Kawahara has been active in is the subject of open boundaries in which linear time-dependent dispersive waves are considered in an unbounded domain. The infinite domain is truncated via an artificial boundary $\mathscr{B}$ on which an open boundary condition (OBC) is imposed. In this paper, Higdon $\mathrm{OBCs}$ and Hagstrom-Hariharan $(\mathrm{HH}) \mathrm{OBCs}$ are considered. Higdontype conditions, originally implemented as low-order $\mathrm{OBCs}$, are made accessible for any desired order via a new scheme. The higher-order Higdon $\mathrm{OBC}$ is then reformulated using auxiliary variables and made compatible for use with finite element (FE) methods. Methodologies for selecting Higdon parameters are also proposed. The performances of these schemes are demonstrated in two numerical examples. This is followed by a discussion of the $\mathrm{HH} \mathrm{OBC}$, which is applicable to non-dispersive media on cylindrical and spherical geometries. The paper extends this OBC to the "slightly dispersive" case.
\end{abstract}

Keywords: Open boundary condition (OBC); Finite element; Dispersive waves; Hagstrom-Hariharan (HH) OBCs

\section{INTRODUCTION}

Among the many areas of research that Professor Kawahara has been active in is the subject of open boundaries. An open boundary is an artificial boundary of a computational domain through which propagating waves or flow should pass in order to leave the computational domain without giving rise to spurious reflection. Open boundaries, which are also called non-reflecting, transparent, absorbing or radiating boundaries, are used in cases where the original domain of the problem under investigation is infinite or very large. Applications include oceanographic and meteorological waves, earthquake waves, air flow around an aircraft and acoustic scattering from submarines. Numerous reviews on the subject are available (Givoli, 1991; 1992; 1999a; Givoli and Harari, 1998; Tsynkov, 1998; Hagstrom, 1999; Turkel, 1998; Astley et al., 2000).
The use of open boundaries is one methodology out of several that have been developed for the numerical solution of exterior wave problems in the last three decades (Givoli, 1992). The 70s and early 80s produced well-known low-order local open boundary conditions (OBCs), e.g. the Engquist-Majda OBCs (Engquist and Majda, 1979) and the Bayliss-Turkel OBCs (Bayliss and Turkel, 1980). The period between the late 80s and mid 90 s has been characterized by the emergence of exact nonlocal OBCs like those based on the Dirichlet-to-Neumann (DtN) map (Keller and Givoli, 1989; Givoli and Keller, 1990), and by the invention of the perfectly matched layer (PML) (Bérenger, 1994).

The method of OBCs can be described as follows. First, the infinite domain is truncated via an artificial boundary $\mathscr{B}$, thus dividing the original domain into a finite computational domain $\Omega$ and a residual infinite domain $D$.

\footnotetext{
*Tel.: + 1-831-656-3478. Fax: + 1-831-656-2355. E-mail: vjvanjool@nps.navy.mil

Tel.: +972-4-829-3814. Fax: +972-4-823-1848. E-mail: givolid@aerodyne.technion.ac.il

Corresponding author. Tel.: +1-831-656-2235. Fax: +1-831-656-2355. E-mail: bneta@nps.navy.mil
} 


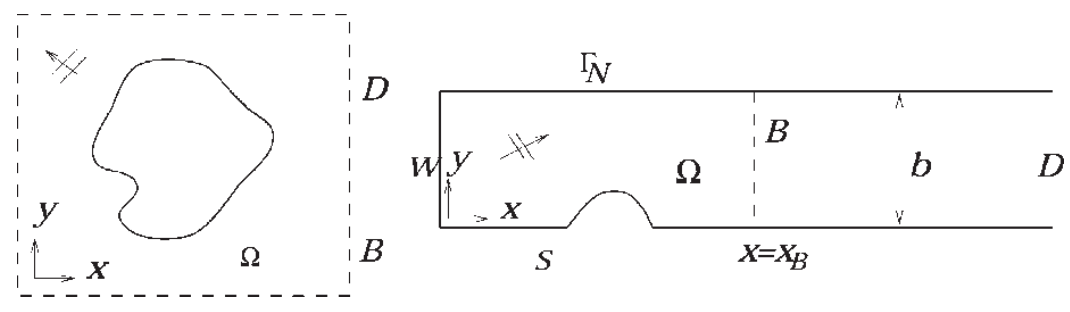

FIGURE 1 Set-up for the OBC method: (a) an exterior scattering problem; (b) a semi-infinite wave guide.

Then a special boundary condition, called an $\mathrm{OBC}$, is imposed on $\mathscr{B}$, in order to complete the statement of the problem in $\Omega$ (i.e. make the solution in $\Omega$ unique) and, most importantly, to ensure that no (or little) spurious wave reflection occurs from $\mathscr{B}$. Finally, the problem is solved numerically in $\Omega$, say by the finite difference (FD) or the finite element (FE) method. The set-up is illustrated in Fig. 1. Fig. 1(a) pertains to an exterior problem outside of a scatterer or an obstacle in full space, whereas Fig. 1(b) describes a semi-infinite wave-guide problem.

Naturally, the quality of the numerical solution strongly depends on the properties of the OBC employed. In the last 25 years, much research was done to develop OBCs that after discretization lead to a scheme which is stable, accurate, efficient and easy to implement (Tsynkov, 1998; Givoli, 1999a; Hagstrom, 1999). Of course, it is difficult to find a single $\mathrm{OBC}$ which is ideal in all respects and all cases; this is why the quest for better OBCs and their associated discretization schemes continues.

Two of Professor Kawahara's investigations on open boundaries are reported by Shimura and Kawahara (1990) and Ohashi and Kawahara (1998). In Shimura and Kawahara (1990), a new boundary treatment is proposed for the two- and three-dimensional (2D and 3D) timedependent incompressible Navier-Stokes equations. The equations are discretized by FEs in space. Iso-parametric linear interpolation functions are used for both pressure and velocity. The authors show that good numerical results are obtained with no need for artificial smoothing. In Shimura and Kawahara (1990), a new OBC is devised for the 2D unsteady non-linear shallow water equations. The numerical solution is matched on the artificial boundary with an analytic solution in a weak manner. Linear triangular FEs are used in the computational domain, and the two-step explicit method is employed as a time integrator.

Sequences of OBCs with increasing order have been available for a long time (e.g. the Bayliss-Turkel conditions [Bayliss and Turkel, 1980] constitute such a sequence), but before the mid 90s they had been regarded as impractical beyond second or third order from the implementation point of view. Recently, practical highorder local OBCs have been introduced (Collino, 1993; Grote and Keller, 1996; Hagstrom et al., 1998; Guddati and Tassoulas, 2000; Givoli, 2001; Givoli and Patlashenko, 2002) that do not involve high-order derivatives. This is enabled by the introduction of special auxiliary variables on $\mathscr{B}$.
In the context of artificial boundary treatment, wave problems can roughly be divided into four categories: (1) linear time-harmonic wave problems, (2) linear timedependent wave problems in non-dispersive homogeneous media, (3) linear time-dependent wave problems in dispersive and/or stratified media and (4) non-linear time-dependent wave problems.

Linear time-harmonic waves have been treated extensively by $\mathrm{OBCs}$ and absorbing layers; see the reviews mentioned above. Time-dependent waves are considerably more difficult to handle from the artificialboundary perspective. However, some exact and highorder schemes have been devised in this case as well. These include the schemes proposed by Collino (1993), Grote and Keller (1996), Hagstrom et al. (1998), Guddati and Tassoulas (2000) and Givoli (2001).

The presence of wave dispersion and/or medium stratification makes the time-dependent problem still more difficult as far as OBC treatment is concerned. Wave dispersion appears in various applications, including meteorological models, which take into account the earth rotation (Pedlosky, 1987; Durran, 1999). None of the high-order and exact OBCs mentioned above has been designed to deal with wave dispersion. In fact, even in one spatial dimension, an exact $\mathrm{OBC}$ for the dispersive (Klein-Gordon) wave equation is not available. Very recently, Navon et al. (2003) developed a PML scheme for the dispersive shallow water equations. Non-linear waves (with the non-linearity extending to infinity) are, of course, the most difficult to handle. Some highly-accurate OBCs have been proposed for specific classes of nonlinear wave problems (Tsynkov, 1998; Givoli, 1999b; Hagstrom, 1999).

This paper describes extension to previous works by Higdon (1994) and Hagstrom et al. (1998) with the goal of solving the linear time-dependent wave problem in a dispersive media. The Higdon OBC is a family of OBCs useful for analyzing dispersive wave problems on Cartesian coordinates, but whose implementation was considered impractical beyond the third order. Methods devised by Givoli and Neta $(2002 ; 2003 a)$ to easily set up and practically employ Higdon OBCs to any order are presented. In doing so, spurious reflection is reduced at the open boundary, however, derivatives beyond the second order result rendering the Higdon condition incompatible with the FE method. Givoli et al. (2003) achieve elimination of all high-order derivatives through 
the introduction of special auxiliary variables on $\mathscr{B}$. This allows the use of any order Higdon OBC while maintaining compatibility with a standard $C^{0} \mathrm{FE}$ formulation, which turns out to be stable even with equal-order interpolation for all the variables. In the second section, we will briefly review the high-order Higdon condition and demonstrate its use with and without auxiliary variables in a Cartesian system. The extension of the Higdon OBC to cylindrical and spherical coordinates is more complicated and still under investigation. The Hagstrom-Hariharan (HH) OBC is useful for analyzing the non-dispersive wave problem in cylindrical- and spherical-coordinates. In the third section, we extend the $\mathrm{HH}$ conditions to the dispersive case. We conclude with remarks for future development.

\section{HIGH-ORDER HIGDON CONDITIONS}

Simply stated the $J$ th-order Higdon $\mathrm{OBC}$ is given by:

$$
H_{J}:\left[\prod_{j=1}^{J}\left(\partial_{t}+C_{j} \partial_{x}\right)\right] \eta=0 \quad \text { on } B \text {. }
$$

Here $\eta$ is the time-dependent dispersive wave under consideration and $C_{j}$ are parameters which must be chosen and which signify phase speeds in the $x$-direction. This equation is exact for all waves that propagate with an $x$-direction phase speed equal to either $C_{1}, \ldots, C_{J}$. The Higdon OBC allows for relatively easy accuracy control, and it can be shown by Higdon (1994) that when a plane wave of the form:

$$
\eta=A \cos \left(\frac{n \pi y}{b}\right) \cos k\left(x-C_{x} t+\psi\right), \quad n=0,1,2 \ldots
$$

impinges on the boundary $\mathscr{B}$ where the Higdon OBC $H_{J}$ is imposed, the resulting reflection coefficient $R$ is:

$$
R=\prod_{j=1}^{J}\left|\frac{C_{j}-C_{x}}{C_{j}+C_{x}}\right|
$$

From Eq. (3) it is evident that by simply increasing the order $J$ of the Higdon OBC, the reflection coefficient $R$ decreases. Theoretically, the boundary condition could be taken to any level of accuracy desired without giving much consideration to the values of $C_{j}$. However, Higdon conditions beyond the third order were considered impractical in terms of implementation and are incompatible with FE schemes. Furthermore, schemes that "intelligently" select values for $C_{j}$ s are often effective in reducing the $\mathrm{OBC}$ order required to achieve a desired level of accuracy. Givoli and Neta (2003a) devise a scheme which discretizes Higdon OBCs of any desired order for implementation with FD schemes. In addition, they utilize a procedure for the automatic choice of the parameters $C_{j}$ using the minimax formula based on the Chebyshev polynomial as proposed by Sommeijer et al. (1986).

\section{Numerical Example Employing Higdon OBC in an FD Formulation}

A uniform wave guide, depicted in Fig. 2 is considered with width $b=5$ and with a dispersion coefficient $f=0.5$. The gravity constant $g=10$ and the layer thickness $\Theta=0.1$, resulting in a medium wave speed $C_{0}=\sqrt{g \Theta}=1$.

The initial values are zero everywhere, and the boundary function $\eta_{\mathrm{W}}$ on the west boundary $\Gamma_{\mathrm{W}}$ is given by:

$$
\begin{aligned}
& \eta_{\mathrm{W}}(y, t) \\
& = \begin{cases}0.005 \cos \left[\frac{\pi}{2 r}\left(y-y_{0}\right)\right] & \text { if }\left|y-y_{0}\right| \leq r \text { and } 0 \leq t \leq t_{0} \\
0 & \text { otherwise. }\end{cases}
\end{aligned}
$$

Thus, the wave source on $\Gamma_{\mathrm{W}}$ is a cosine function in $y$ with three parameters: its center location $y_{0}$, its width $r$ and its time duration $t_{0}$. The chosen parameter values are $y_{0}=2.5, r=1.5$ and $t_{0}=0.5$.

An artificial boundary $\mathscr{B}$ is introduced at $x=5$, thus defining as the computational domain $\Omega$ a $5 \times 5$ square. In $\Omega$ a mesh of $20 \times 20$ is used, with linear interpolation for all the variables. The extended domain for the reference

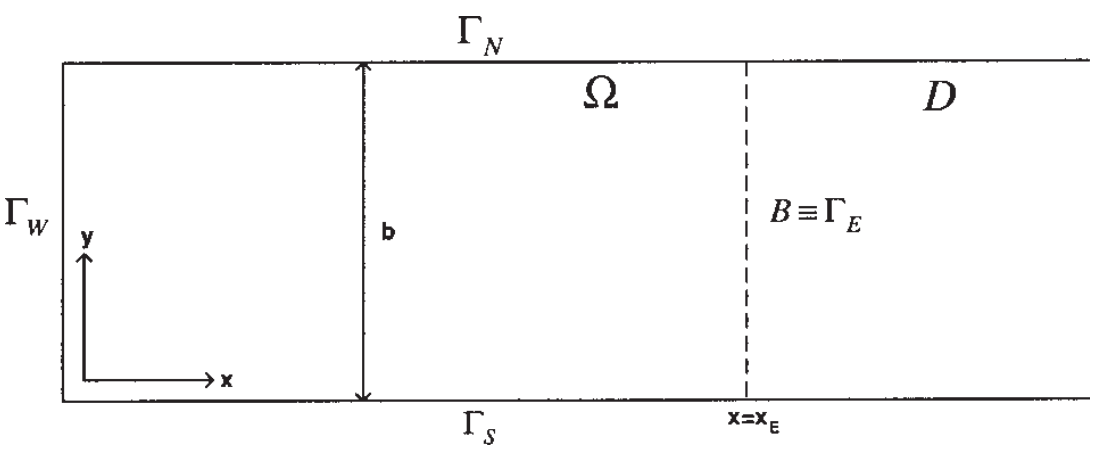

FIGURE 2 Semi-infinite wave guide. 

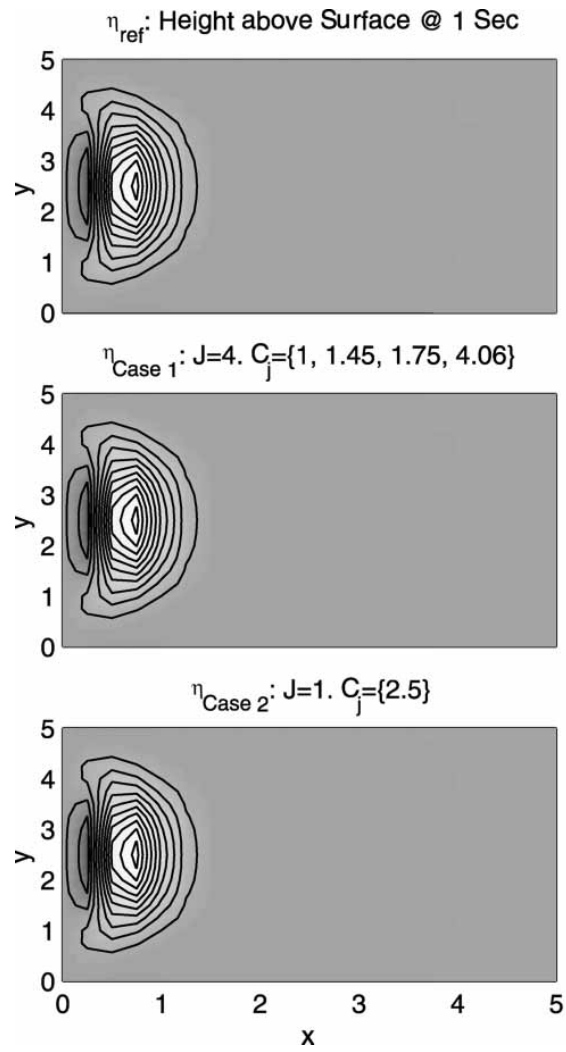

FIGURE 3
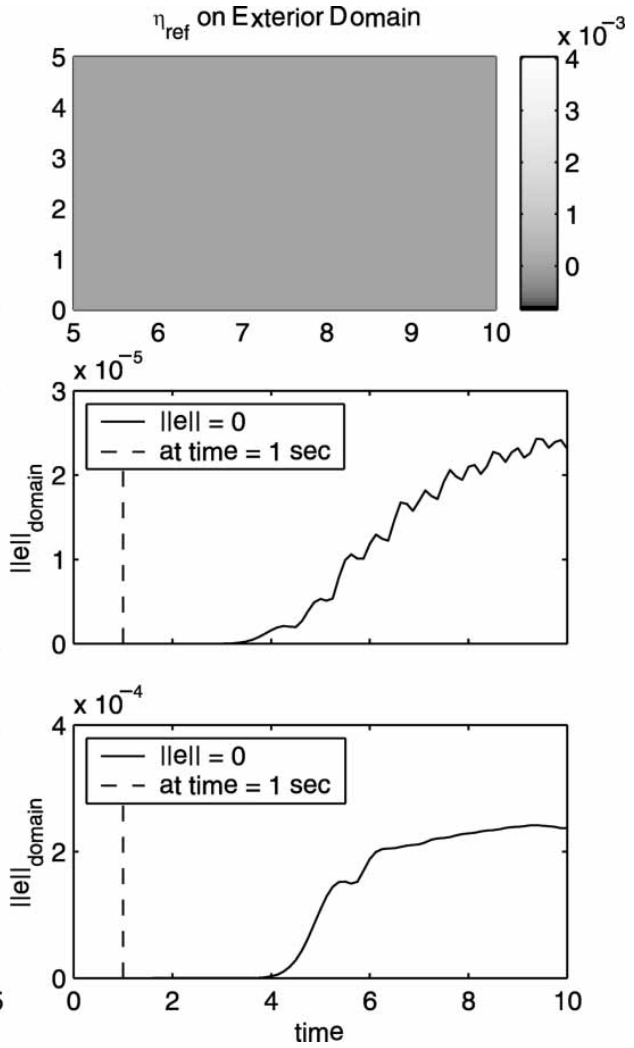

Solution at $1 \mathrm{~s}$

has been separated in the figure so that $\eta_{\text {ref }}$ in $\Omega$ may be better contrasted with $\eta_{\text {case } 1}$ and $\eta_{\text {case } 2}$ ). The middle-left and bottom-left plots correspond to $\eta_{\text {case } 1}$ and $\eta_{\text {case } 2}$, respectively. Two graphs on center- and bottom-right present the $\eta_{\text {case } 1}$ and $\eta_{\text {case } 2}$ error measures that resulted from spurious reflections on $\mathscr{B}$ as a function of time.

At time $t=1$ (Fig. 3) the wave packet $\eta_{\mathrm{W}}$ is still close to $\Gamma_{\mathrm{W}}$ and quite compact. The solution at and near the boundary $\mathscr{B}$ is still zero, hence no spurious reflection has occurred. The plots for $\eta_{\text {ref }}, \eta_{\text {case } 1}$ and $\eta_{\text {case } 2}$ are identical and, as expected, the measured error for $\eta_{\text {case } 1}$ and $\eta_{\text {case } 2}$ is 0 .

At time $t=4$ (Fig. 4) the main bulk of the wave packet just reaches $\mathscr{B}$. A slight spurious reflection is measured for $\eta_{\text {case } 2}$ and $\eta_{\text {case } 1}$, but overall the three solutions in $\Omega$ are still very similar. Note the difference in scales for the vertical axis for the $\eta_{\text {case } 1}$ and $\eta_{\text {case } 2}$ error norm plots indicating that the spurious reflection for the latter case is much greater.

At time $t=5$ (Fig. 5) the front of the wave packet crosses and advances beyond the boundary $\mathscr{B}$. At this time, $\eta_{\text {case } 1}$ is almost indistinguishable from $\eta_{\text {ref }}$, whereas in $\eta_{\text {case 2 }}$ spurious reflections are discernible.

At times $t=8$ and 10 (Figs. 6 and 7), most of the wave packet has left the truncated domain $\Omega$ and is now visible in the extended domain $D$. The solution for $\eta_{\text {case } 1}$ exhibits wave traces which are similar to those present in $\eta_{\text {ref }}$. On the other hand, $\eta_{\text {case } 2}$ reveals a reflected wave that moves backwards in $\Omega$ polluting the computational 

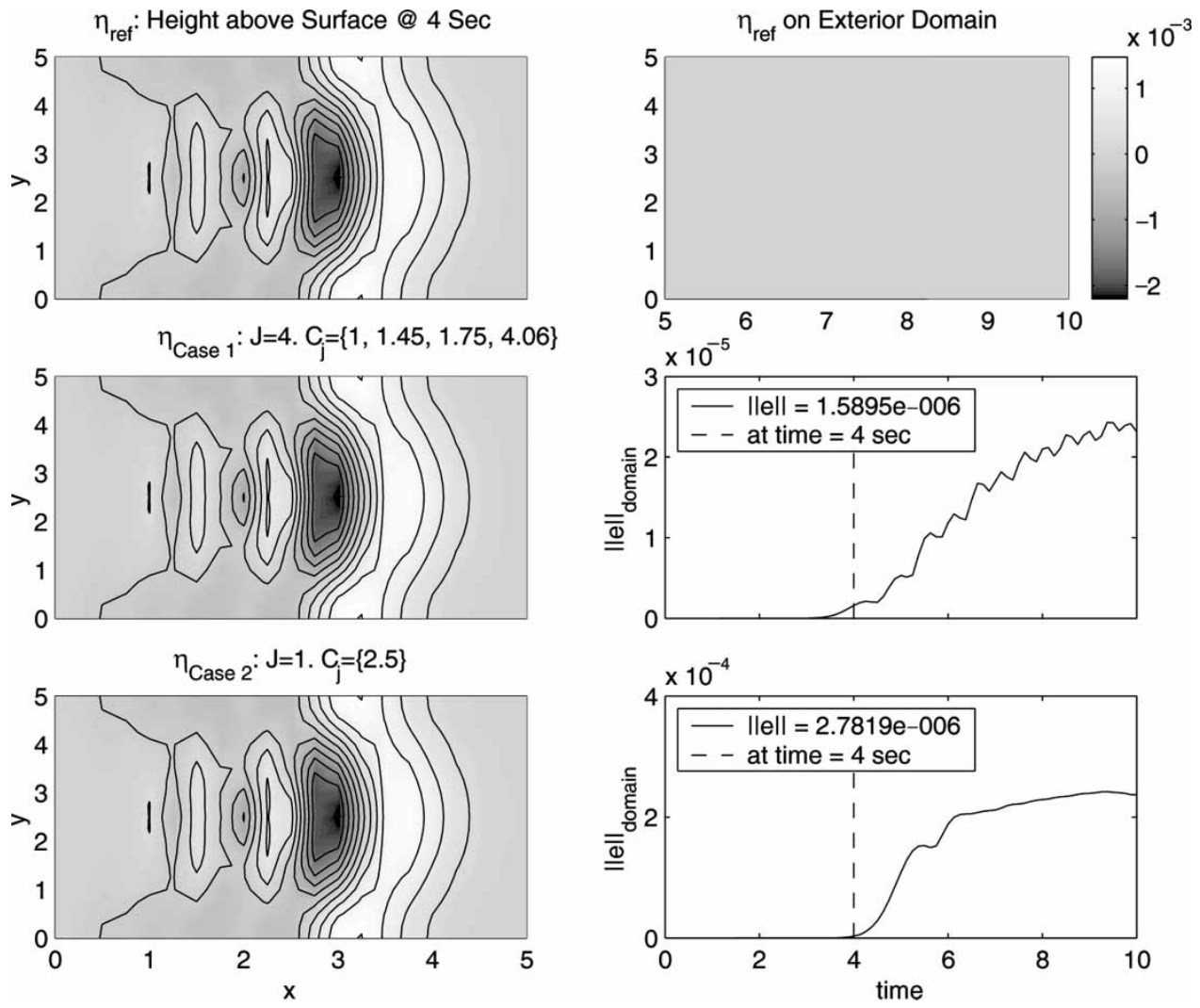

FIGURE 4 Solution at $4 \mathrm{~s}$.
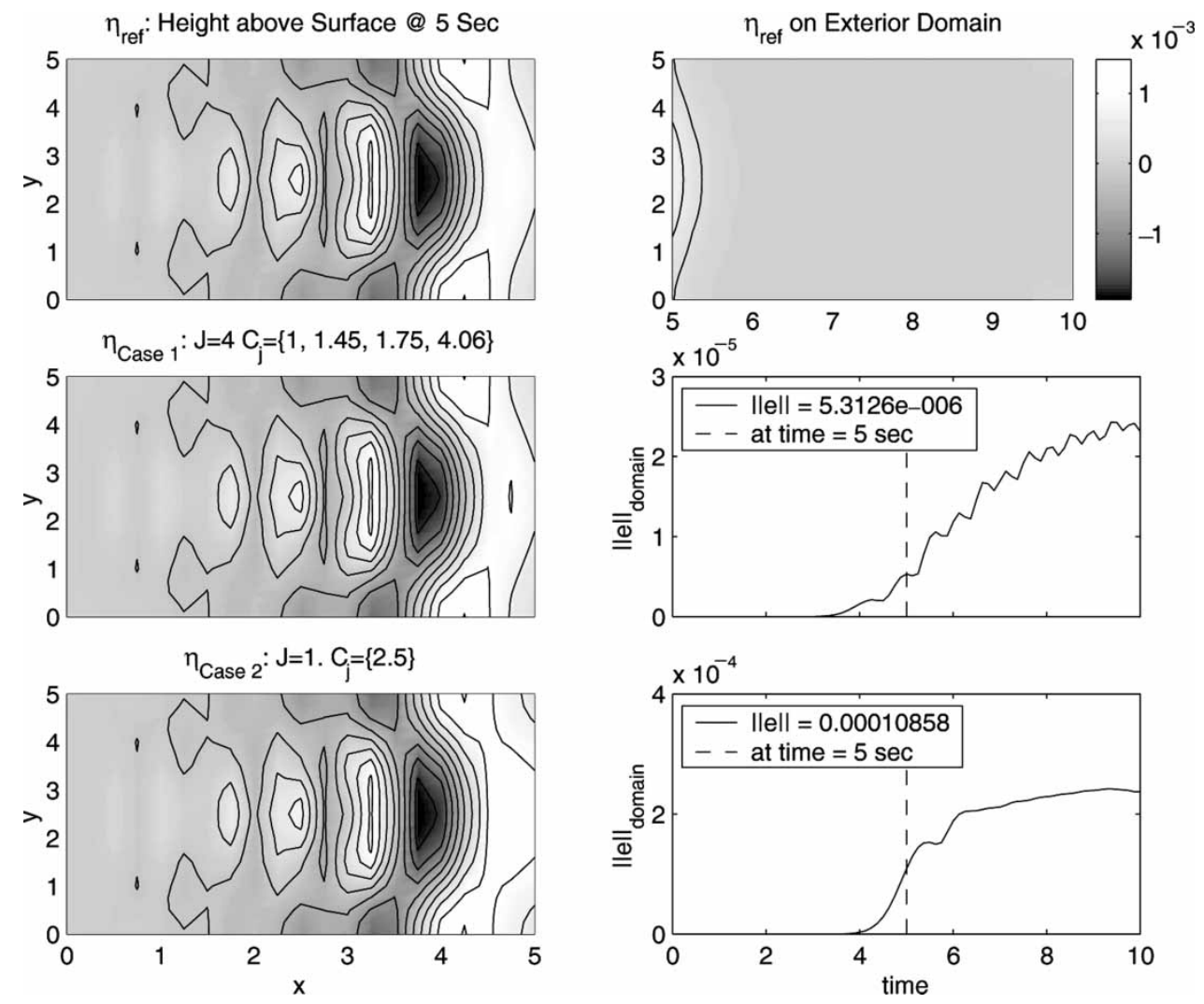

FIGURE 5 Solution at $5 \mathrm{~s}$. 

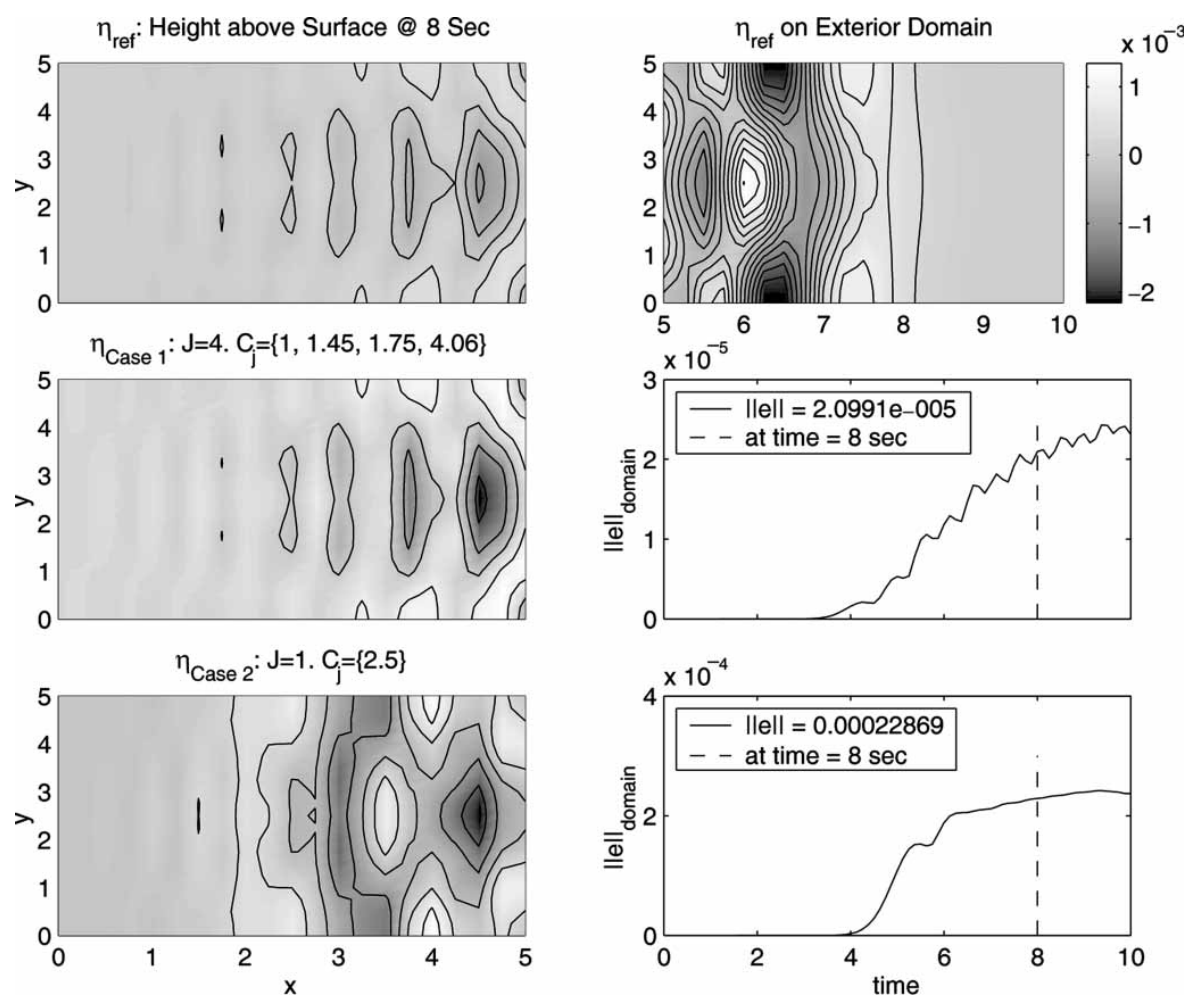

FIGURE 6 Solution at $8 \mathrm{~s}$.
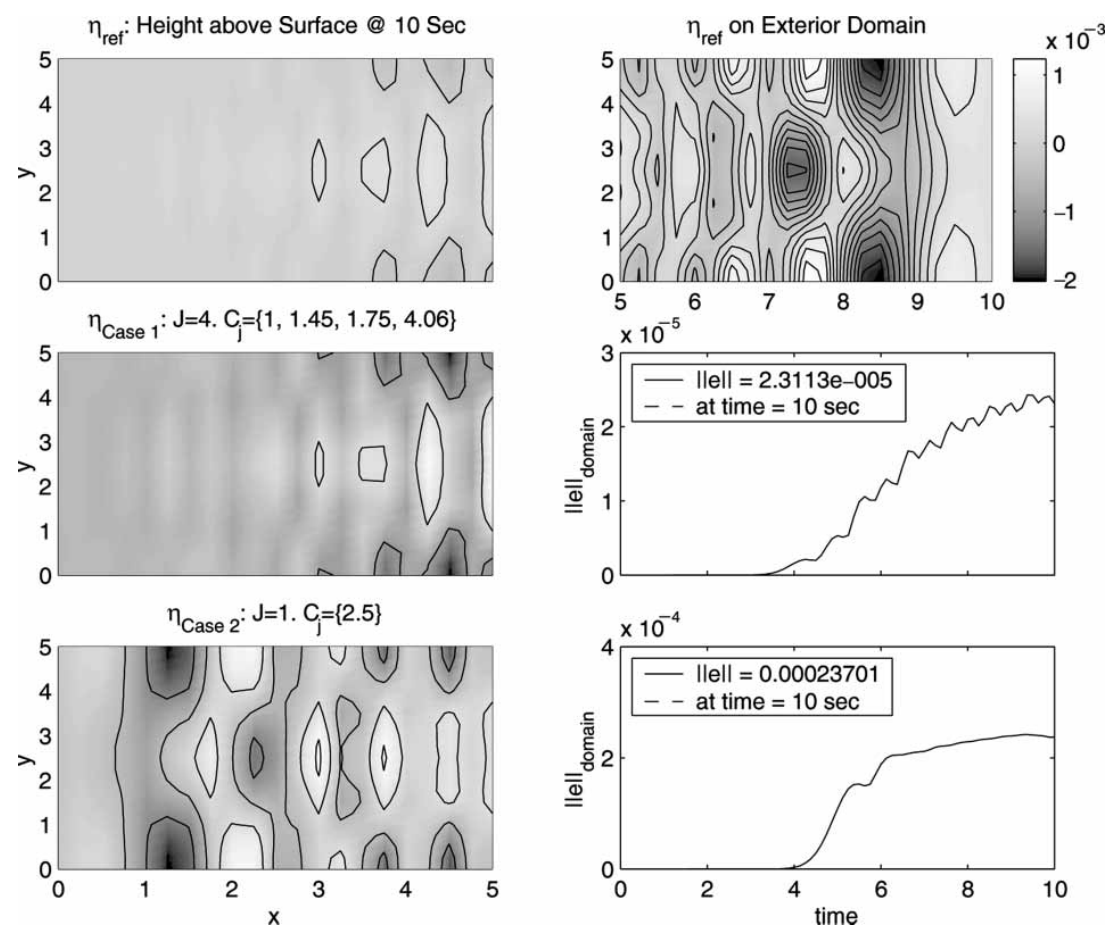

FIGURE 7 Solution at $10 \mathrm{~s}$. 
domain. Again note the difference in scale for the error norm plots which reveal an improvement of one order of magnitude for $\eta_{\text {case } 1}$. This was achieved with minimal computational expense.

\section{Adapting Higher-order Higdon OBCs for Use with FE Methods}

In its current form, Higdon conditions of orders $J=3$ and above are incompatible with FE methods because third-order derivatives or higher are necessary in their formulation. Givoli and Neta (2002) demonstrate how to rewrite the Higdon $\mathrm{OBC}$ with no high-order derivatives by the use of auxiliary variables. As a result, it is more amenable, compared to the previous formulation, for incorporation in an FE scheme, as shown in Givoli et al. (2003).

As a starting point, the Higdon condition given by Eq. (1) is rewritten in an equivalent form:

$$
H_{J}:\left[\prod_{j=1}^{J}\left(\partial_{x}+\frac{1}{C_{j}} \partial_{t}\right)\right] \eta=0 \quad \text { on } \mathrm{B} \text {. }
$$

Auxiliary functions $\phi_{1}, \ldots \phi_{j-1}$, which are defined on $\mathscr{B}$ as well as in the exterior domain outside $\mathscr{B}$ are given as:

$$
\begin{array}{ccc}
\left(\partial_{x}+\frac{1}{C_{1}} \partial_{t}\right) \eta & =\phi_{1} \\
\left(\partial_{x}+\frac{1}{C_{2}} \partial_{t}\right) \phi_{1} & = & \phi_{2} \\
\vdots & \vdots & \vdots \\
\left(\partial_{x}+\frac{1}{C_{J}} \partial_{t}\right) \phi_{J-1} & = & 0 .
\end{array}
$$

By definition these relations hold in $D$ and also in $\mathscr{B}$. If the following definitions are employed:

$$
\phi_{0} \equiv \eta \quad \text { and } \phi_{J} \equiv 0,
$$

Eq. (7) can be concisely written as:

$$
\left(\partial_{x}+\frac{1}{C_{j}} \partial_{t}\right) \phi_{j-1}=\phi_{j}
$$

This set of conditions involves only first-order derivatives. However, due to the appearance of the $x$-derivative in Eq. (9), one cannot discretize the $\phi_{j}$ on the boundary $\mathscr{B}$. Givoli and Neta (2002) manipulate this equation to eliminate the $x$-derivative. The resulting formulation of the Higdon $J$ th-order $\mathrm{OBC}$ on $\mathscr{B}$ is:

$$
\begin{gathered}
\beta_{0} \partial_{t} u+\partial_{x} u=\phi_{1}, \\
\beta_{j} \partial_{t} \phi_{j}-\alpha_{j} \partial_{t}^{2} \phi_{j-1}-\partial_{y}^{2} \phi_{j-1}+\lambda \phi_{j-1}=\phi_{j+1}, \\
\alpha_{j}=\frac{1}{C_{j}^{2}}-\frac{1}{C_{0}^{2}}, \quad \beta_{0}=\frac{1}{C_{1}}, \quad \beta_{j}=\frac{1}{C_{j}}-\frac{1}{C_{j+1}}, \\
\lambda=\frac{f^{2}}{C_{0}^{2}}, \quad \phi_{0} \equiv \eta, \quad \phi_{J} \equiv 0 .
\end{gathered}
$$

This modified form of the Higdon OBC is incorporated into an FE formulation in the numerical example described in the next section. See further discussion of the computational aspects of the scheme in Givoli et al. (2003).

\section{Numerical Example Employing Modified Higdon OBC in an FE Formulation}

Consider the wave-guide problem illustrated in Fig. 8.

The Coriolis parameter $f$ is unity and the initial conditions are zero everywhere except in the strip $0 \leq x \leq 1$ where:

$$
\eta(x, y, 0)=H_{(0,1)}(x), \quad \eta_{t}(x, y, 0)=0 .
$$

Note that the initial values do not depend on $y$, but only on $x$ through the "hat function" $H_{(0,1)}(x)$. Second, the medium wave speed $C_{0}$ is not constant in $\Omega$. But rather

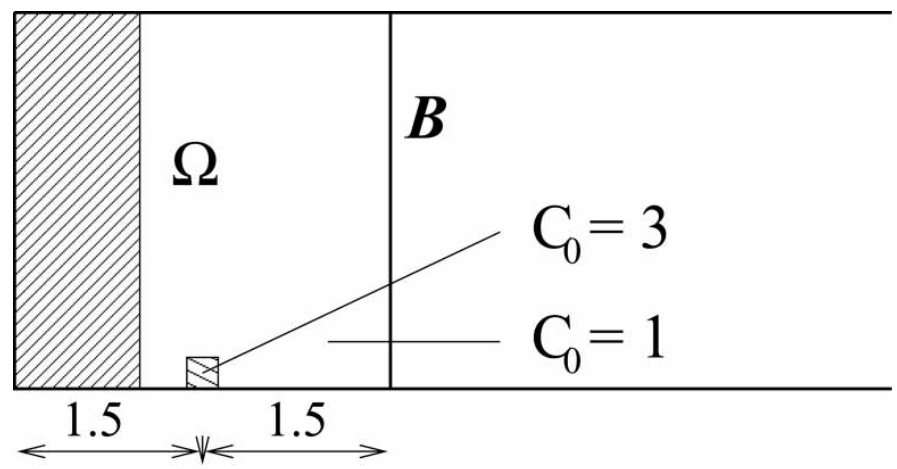

FIGURE 8 Set-up for the "bump" wave-guide problem. 


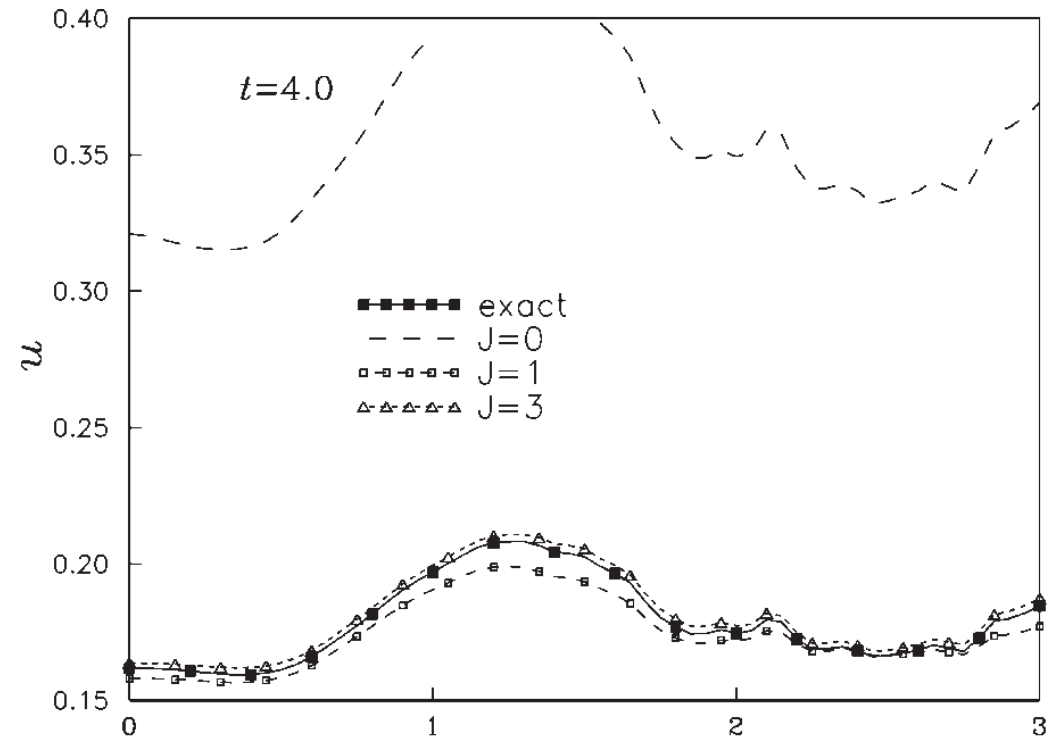

(a) $y$

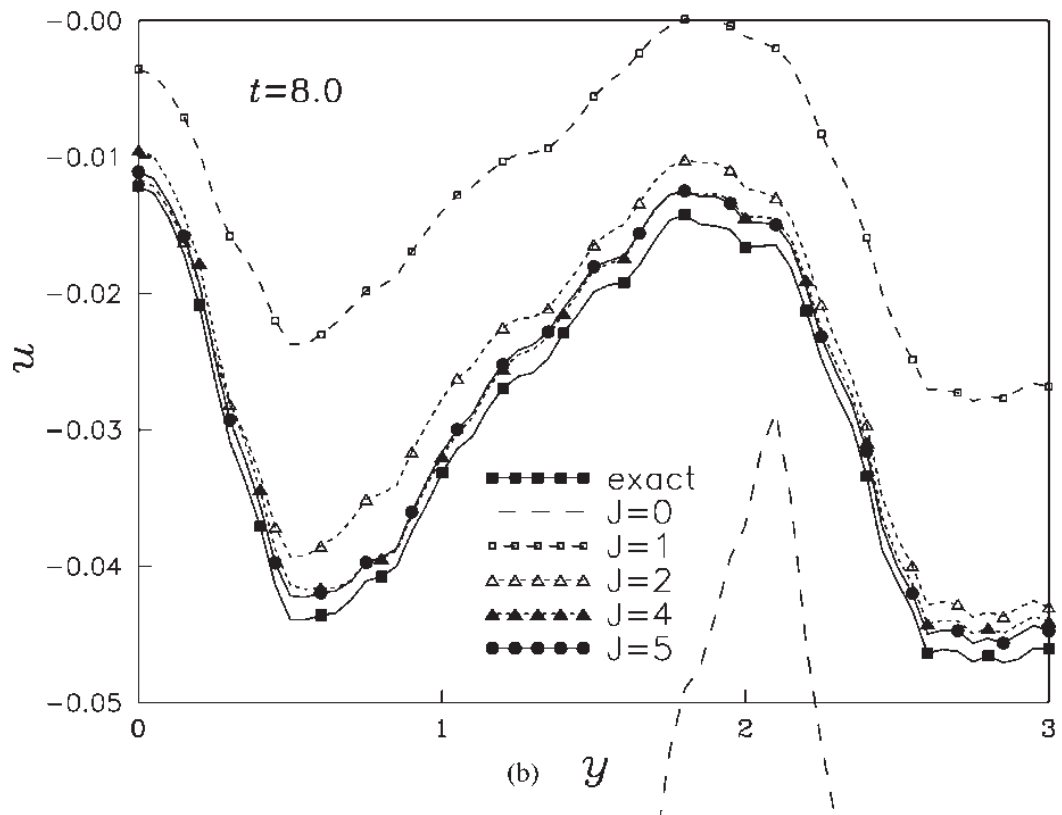

FIGURE 9 The "bump" wave-guide problem: comparison of solutions along the artificial boundary $\mathscr{B}$, at times (a) $t=4$, (b) $t=8$.

$C_{0}=1$ everywhere except in a small square area, of size $0.2 \times 0.2$, where $C_{0}=3$. This area, shown in Fig. 8, models a "hard bump" in the medium, which causes wave scattering inside the wave guide, and is solely responsible for the $y$-dependence of the solution. The "bump" area includes 16 FEs.

Figures 9(a) and (b) show the "exact" solutions as well as solutions obtained for various values of $J$, along $\mathscr{B}$ at times $t=4$ and $t=8$, respectively. The current problem is hard enough so that even with high-order OBCs some small error is noticeable. In particular, note that at time $t=8$ (Fig. 9(b)), the $J=4$ and $J=5$ solutions almost coincide, but are slightly off the "exact" solution. Still, they are much more accurate than the solutions corresponding to $J \leq 3$.
For a given simulation time $T$, one can define the global error-measure in space and time,

$$
\bar{E}_{\mathscr{B}}(T)=\left(\int_{0}^{T} E_{\mathscr{B}}^{2}(t) \mathrm{d} t\right)^{\frac{1}{2}} .
$$

This is the accumulated error on $\mathscr{B}$ during the entire simulation. Figure 10 shows this error as a function of the simulation time $T$ for various values of $J$. For all $J$, the error increases initially with the simulation time, but then becomes almost constant for long simulations. Unlike the instantaneous error shown in the previous figures, the accumulated error decreases monotonically with increasing $J$. The superiority of the $J=5$ solution over all lowerorder solutions is apparent. 


\section{EXTENSION OF THE HAGSTROM-HARIHARAN CONDITIONS TO DISPERSIVE MEDIA}

Hagstrom and Hariharan (1998) devised high-order OBCs for the standard time-dependent 2D wave equation in cylindrical coordinates and for the 3D wave equation in spherical coordinates. The artificial boundary is a circle in $2 \mathrm{D}$ and a sphere in $3 \mathrm{D}$. The $\mathrm{OBC}$ originates from the well-known sequence of conditions of Bayliss and Turkel (1980), but in contrast to them it does not involve any high-order derivatives. This is achieved by employing special auxiliary variables. In Hagstrom and Hariharan (1998), the OBCs are implemented using FDs. Huan and Thompson $(2000 ; 2001)$ implemented the same OBCs with FEs in 3D and 2D, respectively.

Here we shall extend these OBCs to the dispersive case. First, we recall the essential facts from Hagstrom and Hariharan (1998). The standard wave equation in either the $2 \mathrm{D}$-cylindrical or $3 \mathrm{D}$-spherical case is:

$$
\frac{1}{C_{0}^{2}} \frac{\partial^{2} \eta}{\partial t^{2}}=\frac{\partial^{2} \eta}{\partial r^{2}}+\frac{(d-1)}{r} \frac{\partial \eta}{\partial r}+\frac{1}{r^{2}} \tilde{\nabla}_{d}^{2} \eta
$$

Here $d$ is the dimension ( $d=2$ or 3$)$, and

$$
\tilde{\nabla}_{d}^{2} \eta=\left\{\begin{array}{ll}
\frac{\partial^{2} \eta}{\partial \theta^{2}}, & d=2 \\
\frac{1}{\sin \theta} \frac{\partial}{\partial \theta}\left(\sin \theta \frac{\partial \eta}{\partial \theta}\right)+\frac{1}{\sin ^{2} \theta} \frac{\partial^{2} \eta}{\partial \phi^{2}}, & d=3
\end{array} .\right.
$$

The $\mathrm{HH} \mathrm{OBC}$ is based on the following series representation for the solution of Eq. (16):

$$
\text { 2D: } \quad \eta(r, \theta, t) \sim \sum_{n=0}^{\infty} a_{n}(\theta) \sum_{k=0}^{\infty} r^{-\mathrm{k}-1 / 2} f_{k}^{n}\left(C_{0} t-r\right)
$$

$$
\begin{aligned}
3 \mathrm{D}: & \eta(r, \theta, \phi, t) \\
= & \sum_{n=0}^{\infty} \sum_{m=0}^{n} a_{n m}(\theta, \phi) \sum_{k=0}^{\infty} r^{-k-1} f_{k}^{n m}\left(C_{0} t-r\right) .
\end{aligned}
$$

The 3D expansion (19) is a converging series, whereas the $2 \mathrm{D}$ expansion (18) is an asymptotic (non-converging) series valid for large radial distances. Starting from Eqs. (18) and (19), HH obtained the following Pth-order OBC:

$$
\begin{gathered}
\frac{1}{C_{0}} \frac{\partial \eta}{\partial t}+\frac{\partial \eta}{\partial r}+\frac{(d-1)}{2 r} \eta=w_{1}, \\
\frac{1}{C_{0}} \frac{\partial w_{k}}{\partial t}+\frac{k}{r} w_{k}-\frac{\beta_{\mathrm{d} k}}{4 r^{2}} w_{k-1}-\frac{1}{4 r^{2}} \tilde{\nabla}_{d}^{2} w_{k-1} \\
=w_{k+1}, \quad k=1, \ldots, P, \\
\beta_{\mathrm{d} k}= \begin{cases}\left(k-\frac{1}{2}\right)^{2}, & d=2 \\
k(k-1), & d=3\end{cases} \\
w_{0}=2 u, \quad w_{P+1}=0 .
\end{gathered}
$$

The $w_{k}(k=1, \ldots, P)$ are unknown auxiliary functions. Since the $3 \mathrm{D}$ OBC is based on a converging series, it becomes an exact $\mathrm{OBC}$ in the limit $P \rightarrow \infty$. Moreover, in $3 \mathrm{D}$, the $P$ th-order $\mathrm{OBC}$ is exact for all waves consisting of the first $P$ spherical harmonics. The $2 \mathrm{D}$ OBC is only asymptotically correct, for large $R$, where $R$ is the radius of the artificial boundary.

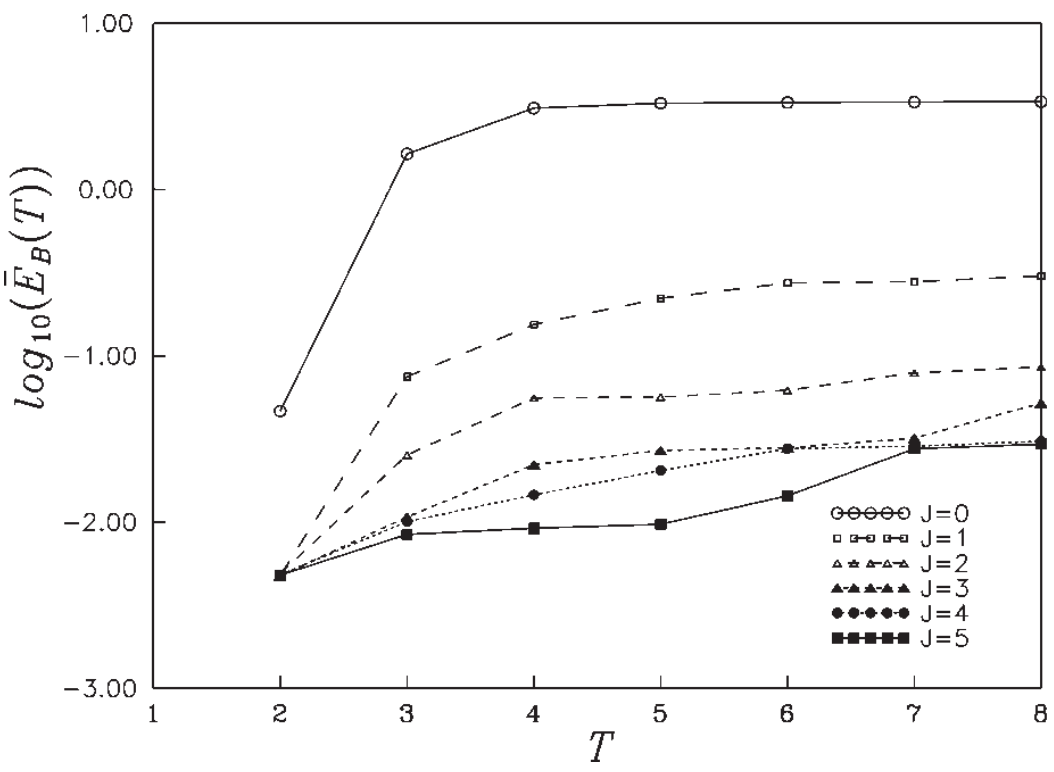

FIGURE 10 The "bump" wave-guide problem: the global accumulated error $\bar{E}_{\mathscr{B}}(T)$ as a function of the simulation time $T$ for various values of $J$. 
Now we consider the dispersive case. In this case, Eq. (16) is replaced by the Klein-Gordon equation:

$$
\frac{1}{C_{0}^{2}} \frac{\partial^{2} \eta}{\partial t^{2}}=\frac{\partial^{2} \eta}{\partial r^{2}}+\frac{(d-1)}{r} \frac{\partial \eta}{\partial r}+\frac{1}{r^{2}} \tilde{\nabla}_{d}^{2} \eta-\frac{f^{2}}{C_{0}^{2}} \eta .
$$

Here $f$ is the dispersion parameter. We shall assume that the dispersion is small, namely that

$$
\frac{f}{C_{0} K} \ll 1
$$

where $K$ is a typical wave number appearing in the solution. This is a common situation in various applications, e.g. meteorology.

In order to extend the $\mathrm{HH}$ conditions $(20)-(23)$ to the dispersive case, we first Fourier-transform them to the frequency domain. This is easily done by formally performing the replacement:

$$
\frac{1}{C_{0}} \frac{\partial}{\partial t} \rightarrow-i K \equiv-i \frac{\omega}{C_{0}} .
$$

Here $K$ is the wave number and $\omega$ is the frequency. This reduces Eqs. (20) and (21) to:

$$
\begin{gathered}
-i K \bar{\eta}+\frac{\partial \bar{\eta}}{\partial r}+\frac{(d-1)}{2 r} \bar{\eta}=\bar{w}_{1}, \\
-i K \bar{w}_{k}+\frac{k}{r} \bar{w}_{k}-\frac{\beta_{\mathrm{d} k}}{4 r^{2}} \bar{w}_{k-1}-\frac{1}{4 r^{2}} \tilde{\nabla}_{d}^{2} \bar{w}_{k-1}=\bar{w}_{k+1}, \\
k=1, \ldots, P .
\end{gathered}
$$

Here a superposed bar indicates a Fourier-transformed variable.

Now, if we Fourier-transform the standard wave equation (16) and the Klein-Gordon equation (24), we obtain the Helmholtz equation in both cases:

$$
\frac{\partial^{2} \bar{\eta}}{\partial r^{2}}+\frac{(d-1)}{r} \frac{\partial \bar{\eta}}{\partial r}+\frac{1}{r^{2}} \tilde{\nabla}_{d}^{2} \bar{\eta}+\hat{K}^{2} \bar{\eta}=0
$$

In the non-dispersive case we have:

$$
\hat{K}=\frac{\omega}{C_{0}} \equiv K,
$$

whereas in the dispersive case we have:

$$
\hat{K}=\frac{\sqrt{\omega^{2}-f^{2}}}{C_{0}}=\sqrt{K^{2}-\frac{f^{2}}{C_{0}^{2}}} .
$$

From Eqs. (30) and (31) we deduce that in the frequency domain, an equation valid in the non-dispersive case becomes valid in the dispersive case under the replacement:

$$
K \rightarrow \sqrt{K^{2}-\frac{f^{2}}{C_{0}^{2}}}
$$

Now we make use of the smallness of $f$ to approximate the square root on the right side of Eq. (32) by a rational function. We use the Taylor approximation:

$$
\sqrt{1-x}=1-\frac{1}{2} x+O\left(x^{2}\right)
$$

to obtain, under the assumption (25),

$$
\sqrt{K^{2}-\frac{f^{2}}{C_{0}^{2}}} \simeq K-\frac{f^{2}}{2 C_{0}^{2} K}
$$

From Eqs. (32) and (34) we get that the replacement:

$$
K \rightarrow K-\frac{f^{2}}{2 C_{0}^{2} K}
$$

which moves us from the non-dispersive case to the slightly dispersive case. Using this we get the dispersive version of Eqs. (27) and (28), namely

$$
\begin{gathered}
-i K \bar{\eta}+\frac{i f^{2}}{2 C_{0}^{2} K} \bar{\eta}+\frac{\partial \bar{\eta}}{\partial r}+\frac{(d-1)}{2 r} \bar{\eta}=\bar{w}_{1}, \\
-i K \bar{w}_{k}+\frac{i f^{2}}{2 C_{0}^{2} K} \bar{w}_{k}+\frac{k}{r} \bar{w}_{k}-\frac{\beta_{\mathrm{d} k}}{4 r^{2}} \bar{w}_{k-1}-\frac{1}{4 r^{2}} \tilde{\nabla}_{d}^{2} \bar{w}_{k-1} \\
=\bar{w}_{k+1}, \quad k=1, \ldots, P .
\end{gathered}
$$

Now it remains to apply the inverse Fourier transform to Eqs. (36) and (37) in order to obtain dispersive OBCs in the time domain. This is formally done by performing the replacements:

$$
i K \rightarrow-\frac{1}{C_{0}} \frac{\partial}{\partial t}, \quad \frac{i}{K} \bar{\eta} \rightarrow C_{0} \int_{0}^{t} \eta(\tau) \mathrm{d} \tau .
$$

In writing the latter formula we have used the fact that the solution vanishes identically on the boundary at time $t=0$. Applying Eq. (38) to Eqs. (36) and (37) finally yields:

$$
\frac{1}{C_{0}} \frac{\partial \eta}{\partial t}+\frac{f^{2}}{2 C_{0}} \int_{0}^{t} \eta(\tau) \mathrm{d} \tau+\frac{\partial \eta}{\partial r}+\frac{(d-1)}{2 r} \eta=w_{1},
$$

$$
\begin{gathered}
\frac{1}{C_{0}} \frac{\partial w_{k}}{\partial t}+\frac{f^{2}}{2 C_{0}} \int_{0}^{t} w_{k}(\tau) \mathrm{d} \tau+\frac{k}{r} w_{k}-\frac{\beta_{\mathrm{d} k}}{4 r^{2}} w_{k-1} \\
-\frac{1}{4 r^{2}} \tilde{\nabla}_{d}^{2} w_{k-1}=w_{k+1}, \quad k=1, \ldots, P,
\end{gathered}
$$

$$
\beta_{\mathrm{d} k}= \begin{cases}\left(k-\frac{1}{2}\right)^{2}, & d=2 \\ k(k-1), & d=3\end{cases}
$$

$$
w_{0}=2 u, \quad w_{P+1}=0 .
$$


By comparing Eqs. (20)-(23) with Eqs. (39)-(42) we see that the effect the dispersion has on the OBCs is in introducing the time-integral terms in Eqs. (39) and (40). We note that these terms do not really make the OBC nonlocal in time, since a single differentiation eliminates the integrals. This does not mean, however, that Eqs. (39) and (40) should be differentiated in practice. From a numerical perspective, despite the appearance of the integral in these equations there is no need to store and operate on the history of the solution. To see this, we denote:

$$
I(t)=\int_{0}^{t} \eta(\tau) \mathrm{d} \tau
$$

Then obviously:

$$
I(t+\Delta t)=I(t)+\int_{t}^{t+\Delta t} \eta(\tau) \mathrm{d} \tau,
$$

and hence the integral I can be calculated in each time-step based on its value in the previous time-step only.

\section{FUTURE WORK}

Several topics for future research remain for both Higdon and $\mathrm{HH} \mathrm{OBCs}$. With regards to the Higdon condition, the authors are currently investigating the extension to cylindrical and spherical geometries. The difficulty is in the fact that the coefficients of the operator $H_{J}$ are not constants. Further investigation is also warranted in techniques that automatically select $C_{j} \mathrm{~s}$ with the goal of minimizing spurious reflections at the artificial boundary while also minimizing the order of the corresponding Higdon OBC. Software routines are also desirable that would employ Higdon OBCs to implement schemes that provide solutions to the non-linear, time-dependent wave equations. With regards to the $\mathrm{HH}$ condition, extending the condition further to the dispersive vice "slightly dispersive" case remains open.

\section{CONCLUSIONS}

In this paper, we discussed the subject of open boundaries in which linear time-dependent dispersive waves are considered in an unbounded domain. The infinite domain is truncated via an artificial boundary \$beta\$ on which an OBC is imposed. In this paper Higdon OBCs and Hagstrom-Hariharan OBCs were considered. Higdon-type conditions, originally implemented as loworder OBCs, are made accessible for any desired order via a new scheme. The higher-order Higdon OBC is then reformulated using auxiliary variables and made compatible for use with Finite Element (FE) methods. The performance of these schemes was demonstrated in two numerical examples. The paper extends the Hagstrom-Hariharan OBC to the "slightly dispersive" case on cylindrical and spherical geometries.

\section{Acknowledgements}

The first author acknowledges the support extended to him by the US Navy and Naval Postgraduate School. The second author would like to express his gratitude for the support extended to him by the National Research Council Associateship Programs. All authors acknowledge the support from ONR (Marine Meteorology and Atmospheric Effects) grant N0001402WR20211 managed by Dr Simon Chang. This work was also supported in part by the Naval Postgraduate School. The authors also thank Dr Igor Patlashenko for permission to use his FE code.

\section{References}

Astley, J., Gerdes, K., Givoli, D. and Harari, I. eds. (2000) "Special Issue", J. Comput. Acoust. 8(1).

Bayliss, A. and Turkel, E. (1980) "Radiation boundary conditions forods wave-like equations", Comm. Pure Appl. Math. 33, $707-725$.

Bérenger, J.P. (1994) "A perfectly matched layer for the absorption of electromagnetic waves", J. Comput. Phys. 114, 185-200.

Collino, F., et al. (1993) "High order absorbing boundary conditions for wave propagation models. Straight line boundary and corner cases", In: Kleinman, R., ed, Proceedings of 2nd International Conference on Mathematical \& Numerical Aspects of Wave Propagation (SIAM, Delaware) pp 161-171.

Durran, D.R. (1999) Numerical Methods for Wave Equations in Geophysical Fluid Dynamics (Springer, New York).

Engquist, B. and Majda, A. (1979) "Radiation boundary conditions for acoustic and elastic calculations", Comm. Pure Appl. Math. 32, 313-357.

Givoli, D. (1991) "Non-reflecting boundary conditions: a review", J. Comput. Phys. 94, 1-29.

Givoli, D. (1992) Numerical Methods for Problems in Infinite Domains (Elsevier, Amsterdam).

Givoli, D. (1999a) "Exact representations on artificial interfaces and applications in mechanics", Appl. Mech. Rev. 52, 333-349.

Givoli, D. (1999b) "Recent advances in the DtN finite element method for unbounded domains", Arch. Comput. Methods Eng. 6, $71-116$.

Givoli, D. (2001) "High-order non-reflecting boundary conditions without high-order derivatives", J. Comput. Phys. 170, $849-870$.

Givoli, D. and Harari, I. (1998) "Exterior Problems of Wave Propagation, Special Issue", Comput. Methods Appl. Mech. Eng. 164(1-2).

Givoli, D. and Keller, J.B. (1990) "Non-reflecting boundary conditions for elastic waves", Wave Motion 12, 261-279.

Givoli, D. and Neta, B. (2002) High-Order Higdon Non-Reflecting Boundary Conditions for the Shallow Water Equations Rep. No. NPSMA-02-001 (Naval Postgraduate School, Monterey, CA).

Givoli, D. and Neta, B. (2003) "High-order non-reflecting boundary conditions for dispersive waves", Wave Motion 37, 257-271.

Givoli, D. and Neta, B. (2003) "High-order non-reflecting boundary scheme for time-dependent waves", J. Comput. Phys. 186, 24-46.

Givoli, D. and Patlashenko, I. (2002) "An optimal high-order non-reflecting finite element scheme for wave scattering problems", Int. J. Numer. Methods Eng. 53, 2389-2411.

Givoli, D., Neta, B. and Patlashenko, I. (2003) "Finite element analysis of time-dependent semi-infinite wave-guides with high-order boundary treatment", Int. J. Numer. Methods Eng., In press.

Grote, M.J. and Keller, J.B. (1996) "Nonreflecting boundary conditions for time dependent scattering", J. Comput. Phys. 127, 52-65.

Guddati, M.N. and Tassoulas, J.L. (2000) "Continued-fraction absorbing boundary conditions for the wave equation", J. Comput. Acoust. 8, $139-156$. 
Hagstrom, T. (1999) "Radiation boundary conditions for the numerical simulation of waves", Acta Numer. 8, 47-106.

Hagstrom, T. and Hariharan, S.I. (1998) "A formulation of asymptotic and exact boundary conditions using local operators", Appl. Numer. Math. 27, 403-416.

Higdon, R.L. (1994) "Radiation boundary conditions for dispersive waves", SIAM J. Numer. Anal. 31, 64-100.

Huan, R. and Thompson, L.L. (2000) "Accurate radiation boundary conditions for the time-dependent wave equation on unbounded domains", Int. J. Numer. Methods Eng. 47, 1569-1603.

Huan, R. and Thompson, L.L. (2001) "Accurate radiation boundary conditions for the two-dimensional wave equation on unbounded domains", Comput. Methods Appl. Mech. Eng. 191, 331-351.

Keller, J.B. and Givoli, D. (1989) "Exact non-reflecting boundary conditions", J. Comput. Phys. 82, 172-192.

Navon, I.M., Neta, B. and Hussaini, M.Y. (2003) "A Perfectly Matched Layer Approach to the Linearized Shallow Water Equations Models", submitted.
Ohashi, T. and Kawahara, M. (1998) "Investigation of open boundary treatment using hybrid method and finite element method", In: Davis, G.D.V. and Leonardi, E., eds, Proceedings of International Symposium on Advances in Computational Hear Transfer, Cesme, Turkey, May 1997 (Begell House Inc. New York, NY).

Pedlosky, J. (1987) Geophysical Fluid Dynamics (Springer, New York). Shimura, M. and Kawahara, M. (1990) "A finite element flow analysis on the open boundary problems", In: Yasuhara, M., Daiguji, H. and Oshima, K., eds, Numerical Methods in Fluid Dynamics II, Proceedings of the International Symposium on CFD, Nagoya, Japan, Aug. 1989 (Japan Society of CFD, Tokyo, Japan).

Sommeijer, B.P., van der Houwen, P.J. and Neta, B. (1986) "Symmetric linear multistep methods for second order differential equation with periodic solutions", Appl. Numer. Math. 2, 69-77.

Tsynkov, S.V. (1998) "Numerical solution of problems on unbounded domains, a review", Appl. Numer. Math. 27, 465-532.

Turkel, E., ed. (1998) Appl. Numer. Math., Special Issue, Vol. 27. 